\title{
Shining light of physics
}

\section{N. Straumann}

The Collected Papers of Albert Einstein. Volume 2: The Swiss Years: Writings, 1900-1909. Edited by John Stachel. Princeton University Press: 1990. Pp.656. \$85. (English translation by Anna Beck available separately. Pp.398. \$25.)

THIS second volume of Einstein's Collected Papers might well turn out to be the most important of the whole projected series of approximately 30 volumes. It contains Einstein's scientific work during the first decade of his career, beginning with two papers on the nature of intermolecular forces and ending with his famous Salzburg lecture "On the Development of our Views Concerning the Nature and Constitution of Radiation".

This beautiful volume contains 62 annotated documents presented in facsimile, 8 well-written editorial notes and a useful introduction. By contrast to the first volume, there are no untranslated German quotations in the editorial notes and annota- Einstein - establishing a central role in modern physics. tions. An accompanying volume contains the English translations of the documents, all of which were originally written in German. People who know only English should have no problem in reading these in conjunction with the documentary edition and its editorial apparatus.

It is well known that in March, May and June of 1905, Einstein wrote the three masterpieces on three widely different fields of physics which established him as a central figure in twentieth-century physics. But even before that year of miracles, he had published several remarkable papers on the problems of molecular reality and the molecular basis of thermodynamics. In particular, his work on statistical mechanics was absolutely crucial for his most revolutionary contribution to physics: the light-quantum hypothesis. It may be recalled that Einstein extracted the light-quantum postulate from an analogy between radiation in the Wien regime and a classical ideal gas of material particles. In doing so, Boltzmann's principle and Einstein's understanding of statistical fluctuations played an important role.
Einstein gave to the physics section of the Gesellschaft Deutscher Naturforscher und
Einstein wrote three papers devoted exclusively to the foundations of statistical mechanics (documents 3-5), in which he tried to fill what he considered to be a gap in the mechanical foundations of thermodynamics. The harvest of results used in his later work is well described in one of the editorial notes here. When Einstein wrote his three papers he was not familiar with the work of Gibbs (or of some of Boltzmann's). Einstein's papers form a bridge, parallel to that of Gibbs in 1902, between Boltzmann's work and the modern approach to statistical mechanics. In particular, Einstein independently formulated the distinction between the microcanonical and canonical ensembles

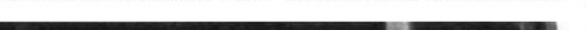

Aerzte at its 1909 meeting in Salzburg. Pauli once said that this report can be regarded as a turning-point in the development of theoretical physics. Einstein discussed the theory of relativity and quantum theory and pointed out important interconnections between his work on the quantum hypothesis, on relativity, and on brownian motion and statistical physics. In particular, he analysed fluctuations in the energy and momentum of the radiation field, drawing on ideas and methods he had developed earlier in the course of his work on statistical physics and brownian motion. Einstein maintained that "the next phase in the development of theoretical physics will bring us a theory of light that may be conceived as a sort of fusion of the wave and of the emission theory of light". In this talk he gave the first synthesis of the profound transformation in the concept of light brought about by the theory of relativity and the quantum hypothesis, and of the profound implications of this transformation for the development of physics.

Many scientists will read again and again this long-awaited volume, which contains some of the most significant achievements of twentieth-century physics.

N. Straumann is Professor of Theoretical Physics, University of Zurich, $\mathrm{CH}-8001$ Zurich, Switzerland.

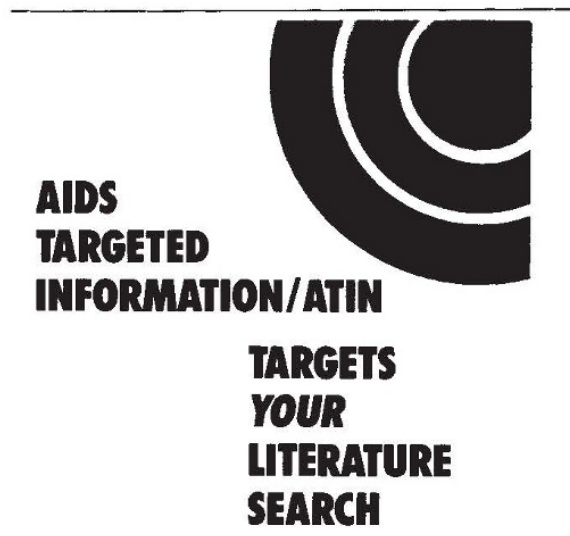

Expand your base of knowledge about

AIDS/HIV with AIDS Targeted

INformation/ATIN. Every month, ATIN

not only provides abstracts but also in-

depth evaluations of the current published

scientific literature on AIDS. Written by

clinicians and researchers for clinicians

and researchers, ATIN provides an

authoritative command of the world's

literature on AIDS. Published by Williams

\& Wilkins. Indexed. 12 issues per year.

$\$ 125$.

\section{To order call \\ $1 \cdot 800 \cdot 638 \cdot 6423$}

Sponsored by the American Foundation for AIDS Research (AmFAR). 